\title{
Analytical Model of an I-core Coil for Nondestructive Evaluation of a Conducting Cylinder below an Infinite Plane Conductor
}

\author{
Siquan Zhang \\ Department of Electrical and Automation, Shanghai Maritime University, 1550 Haigang Avenue, Pudong District, 201306, \\ Shanghai, China, sqzhang@shmtu.edu.cn
}

\begin{abstract}
An analytical model for eddy current testing of an I-core coil located above a two-layer conductive material is presented. The upper layer is an infinite plane conductor, and the bottom layer is a conductive cylinder. The method of truncated region eigenfunction expansion (TREE) is used to solve this axisymmetric problem. First the magnetic vector potential of a filamentary coil coaxial with the I-core over the twolayer conductor is considered. Then the closed form expression for the impedance of the multi-turn coil with rectangular cross section is derived by using the principle of superposition from the filamentary coil field. For frequencies ranging from $0.1 \mathrm{kHz}$ to $10 \mathrm{kHz}$, both the impedance changes of the I-core coil located above the infinite plane conductor without the conducting cylinder, and in the absence of the two-layer conductor are calculated using Mathematica, respectively. The influence of the conducting cylinder below the infinite plane conductor on the impedance change is analyzed. The analytical calculation results are verified by the finite element method and experiment, the results agree very well, which verifies the correctness of the analytical model.
\end{abstract}

Keywords: Analytical model, coil impedance, eddy current testing, truncated region eigenfunction expansion, conductive cylinder.

\section{INTRODUCTION}

Eddy current testing (ECT) is a nondestructive method used for detection of surface and subsurface defects of conducting material. In addition, eddy current probes have many applications in practice, such as material characteristics evaluation, coating thickness measurement of conductor, or they are directly applied to detect buried defect in multilayered conductor [1], [2]. Usually, a coil of rectangular cross-section surrounds a coaxial cylindrical ferrite core to obtain a higher flux, the flux concentration can achieve the effect of improving the sensitivity of the probe. In order to use the changes of coil impedance measured in ECT to perform inversion algorithm for obtaining accurate conductive material characteristics or defect shape in conductor, it is necessary to derive an analytical model that can realize the simulation of the measurement process and calculate the coil impedance accurately.

The truncated region eigenfunction expansion (TREE) method truncates the solution region in one coordinate dimension and results in an approximate expression of the unbounded domain solution, but the error introduced by truncation can be reduced by increasing the width of the region. The integral solution in the infinite region converts into a series solution in the truncated region [3], [4]. Due to the advantages of fast calculation speed and adjustable solution accuracy, the TREE method has been used in various eddy current analytical models. The models of I-core, C-core, and E-core probes applying the TREE method used for evaluation of layered conducting half-space were presented in [5]-[7]. Analytical models of I-core and E-core coils located above a multi-layer conductor containing cylindrical air hole were obtained in [8]-[10]. However, the analytical problem of a ferrite cored-coil located above a small size conductor such as a conductive cylinder is rarely discussed. But sometimes we may encounter problems such as detecting the existence of a small size conductor and identifying its material properties. A model for coin validation using an aircore coil was presented in [11], and the final expression of impedance change was obtained by superposing the field from the isolated coil and the field from the coin. But this method is not suitable for solving the problem of ferrite-cored coil located above the conductor, because the field of core will be affected by the eddy current in the conductor [11], [12].

As shown in Fig.1. a multi-turn I-core coil with a rectangular cross section is placed over a two-layer structure composed of an upper infinite plane conductor and a bottom conducting cylinder. The TREE method is used to solve this I-core coil impedance calculation problem, and finally the matrix form coil impedance expression is obtained. The coil impedances are calculated in Mathematica and are in good agreement with the results of finite element method and experiments. 


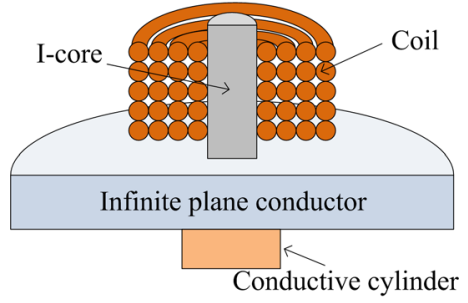

Fig.1. Cross-sectional view of an I-core coil located above an infinite plane conductor and a conductive cylinder.

In Fig.2. the I-core coil is located above a top-layer infinite plane conductor with permeability $\mu_{5}$ and conductivity $\sigma_{5}$, a second-layer conductive cylinder with permeability $\mu_{6}$ and conductivity $\sigma_{6}$. The thickness of the infinite plane conductor is $d_{2}-d_{1}$. The radius of the conducting cylinder is $c$ and the thickness is $d_{3}-d_{2}$. The coil height is $z_{2}-z_{1}$, with an inner radius of $r_{1}$ and an outer radius of $r_{2}$. The I-core has a relative permeability of $\mu_{f}$, a radius of $a_{1}$, and a height of $h_{1}$. Cylindrical coordinate system is adopted, and the radius of the truncated region is $b$. The whole problem region is divided into seven regions along the axial direction. According to Dirichlet boundary conditions, the discrete eigenvalues of each region need to be calculated to determine the expression of magnetic vector potential of each region. The components of magnetic vector potential disappear at $r=0$ and $r=b$ must be satisfied.

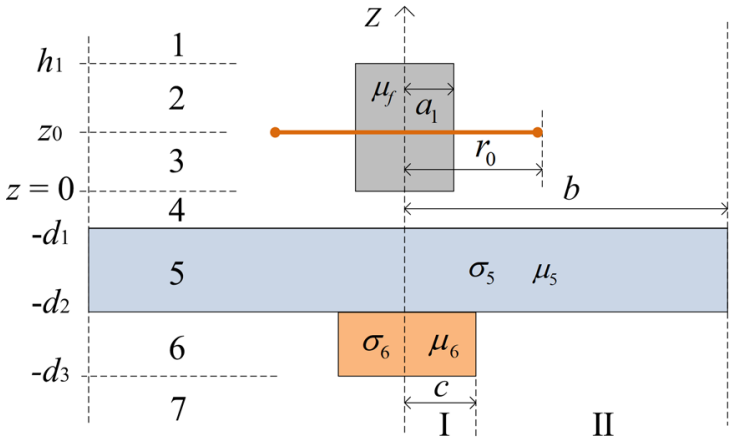

a)

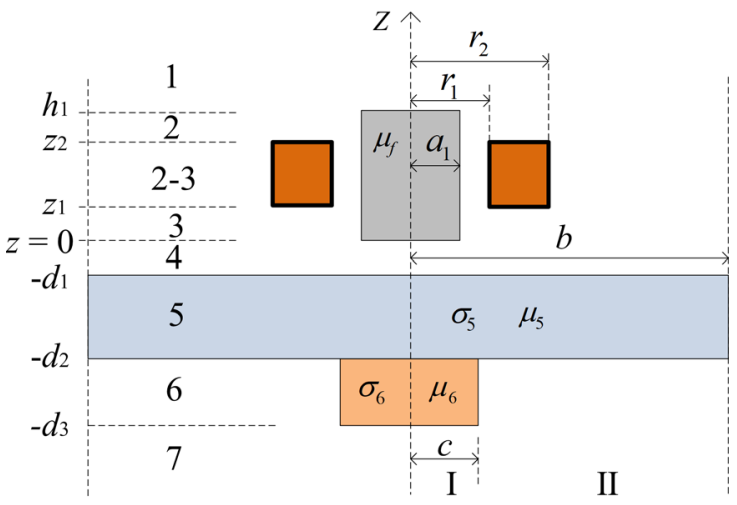

b)

Fig.2. a) filamentary and Fig.2.b) multi-turn axially symmetric Icore coil located above an infinite plane conductor with a conductive cylinder below.

\section{THEORY ANALYSIS}

The single-turn coil, as shown in Fig.2.a), was analyzed first. The eigenvalues of each region were defined as follows.

The eigenvalues $q_{\mathrm{i}}$ of regions $1,4,5$, and 7 are the positive real roots of the following equation:

$$
J_{1}\left(q_{i} b\right)=0, \quad i=0,1,2, \ldots, N s
$$

Because regions 2 and 3 comprise two sub-regions, the magnetic core and the air, the radial dependence in the expressions for these two sub-regions can be written as below:

$$
\begin{gathered}
A_{\text {core }}=A_{E} J_{1}\left(p_{i} r\right) \quad 0 \leq r \leq a_{1} \\
A_{\text {air }}=A_{E} B_{1 F} J_{1}\left(p_{i} r\right)+A_{E} C_{1 F} Y_{1}\left(p_{i} r\right) \quad a_{1} \leq r \leq b
\end{gathered}
$$

Where $p_{i}$ are the corresponding discrete eigenvalues. In regions 2 and 3 , the continuity of $B_{\mathrm{r}}$ and $H_{\mathrm{z}}$ on the interface $r$ $=a_{1}$ yields the following expressions for $B_{1 \mathrm{~F}}$ and $C_{1 \mathrm{~F}}$ :

$$
\begin{gathered}
B_{1 F}=\frac{\pi p_{i} a_{1}}{2}\left[J_{1}\left(p_{i} a_{1}\right) Y_{0}\left(p_{i} a_{1}\right)-\frac{J_{0}\left(p_{i} a_{1}\right) Y_{1}\left(p_{i} a_{1}\right)}{\mu_{f}}\right] \\
C_{1 F}=\frac{\pi p_{i} a_{1}}{2} J_{1}\left(p_{i} a_{1}\right) J_{0}\left(p_{i} a_{1}\right)\left(\frac{1}{\mu_{f}}-1\right)
\end{gathered}
$$

Since at the boundary $r=b, A_{\varphi}(b, z)=0$ must also hold, the following equation is formed:

$$
R_{1}\left(p_{i} b\right)=B_{1 F} J_{1}\left(p_{i} b\right)+C_{1 F} Y_{1}\left(p_{i} b\right)=0
$$

The eigenvalues $p_{i}$ are the real positive roots of (6).

Region 6 contains two sub-regions. Sub-region I $(0 \leq r \leq c)$ contains conductive material, and sub-region II $(c \leq r \leq b)$ contains air. The magnetic vector potentials of these two subregions can be expressed in the following form:

$$
\begin{array}{ll}
A_{I}=A_{E} J_{1}\left(v_{i} r\right) F_{1}\left(u_{i} c\right) & 0 \leq r \leq c \\
A_{I I}=A_{E} J_{1}\left(v_{i} c\right) F_{1}\left(u_{i} r\right) & c \leq r \leq b
\end{array}
$$

where

$$
F_{n}\left(u_{i} r\right)=J_{n}\left(u_{i} r\right) Y_{1}\left(u_{i} b\right)-J_{1}\left(u_{i} b\right) Y_{n}\left(u_{i} r\right)
$$

The relationship between $u_{i}$ and $v_{i}$ is

$$
u_{i}=\sqrt{v_{i}^{2}+j \omega \mu_{0} \mu_{6} \sigma_{6}}
$$

where $J_{n}$ and $Y_{n}$ are Bessel functions of order $n$.

At the radial interface $r=c$, the following equation can be obtained according to the interface conditions:

$$
\frac{1}{\mu_{6}} v_{i} F_{1}\left(u_{i} c\right) J_{0}\left(v_{i} c\right)=u_{i} J_{1}\left(v_{i} c\right) F_{0}\left(u_{i} c\right)
$$


Calculation of eigenvalues $u_{i}$ and $v_{i}$ is reduced to finding complex roots of (11). Using numerical procedures, such as FindRoot[ ] in Mathematica or fzero( ) in Matlab, requires providing initial values around which the roots are located. Such initial values may be obtained upon the assumption that sub-regions I and II consist only of air. Unfortunately, this method may lead to omission of some roots. Higher efficiency can be achieved using the Newton-Raphson technique or adopting more complex algorithms [13], [14].

Following the separation of variables, the expressions for $A_{\varphi}$ in the various regions of the problem in Fig.2.a) have the following form which is given in matrix notation:

$$
\begin{aligned}
& A_{1}(r, z)=J_{1}\left(\mathbf{q}^{\mathbf{T}} r\right) \mathbf{q}^{-1} e^{-\mathbf{q} z} \mathbf{C}_{1}
\end{aligned}
$$

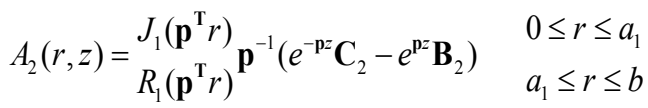

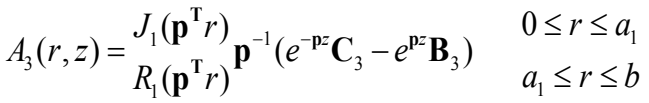

$$
\begin{aligned}
& A_{4}(r, z)=J_{1}\left(\mathbf{q}^{\mathbf{T}} r\right) \mathbf{q}^{-1}\left(e^{-\mathbf{q} z} \mathbf{C}_{4}-e^{\mathbf{q} z} \mathbf{B}_{4}\right) \\
& A_{5}(r, z)=J_{1}\left(\mathbf{q}^{\mathbf{T}} r\right) \mathbf{m}^{-1}\left(e^{-\mathbf{m} z} \mathbf{C}_{5}-e^{\mathbf{m} z} \mathbf{B}_{5}\right)
\end{aligned}
$$

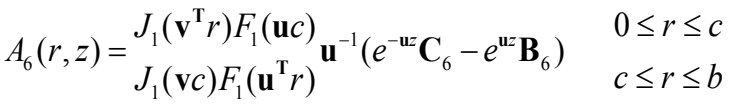

$$
\begin{aligned}
& A_{7}(r, z)=J_{1}\left(\mathbf{q}^{\mathbf{T}} r\right) \mathbf{q}^{-1} e^{\mathbf{q} z} \mathbf{B}_{7}
\end{aligned}
$$

where

$$
\mathbf{m}=\sqrt{\mathbf{q}^{2}+j \omega \sigma_{5} \mu_{0} \mu_{5}}
$$

$J_{1}\left(\mathbf{q}^{\mathrm{T}} r\right), J_{1}\left(\mathbf{p}^{\mathrm{T}} r\right), R_{1}\left(\mathbf{p}^{\mathrm{T}} r\right), J_{1}\left(\mathbf{v}^{\mathrm{T}} r\right), F_{1}\left(\mathbf{u}^{\mathrm{T}} r\right)$ are row vectors. $\mathbf{q}^{-1}, \mathbf{p}^{-1}, \mathbf{m}^{-1}, \mathbf{u}^{-1}, J_{1}(\mathbf{v} c), F_{1}(\mathbf{u} c)$ and exponents $e^{ \pm \mathbf{q} z}, e^{ \pm \mathrm{pz}}, e^{ \pm \mathbf{m} z}$, $e^{ \pm u z}$ are diagonal matrices. $\mathbf{C}_{i}$ and $\mathbf{B}_{i}$ are column vectors of unknown coefficients.

In the filamentary coil problem shown in Fig.2.a), the interface conditions between the seven regions (the continuity of $B_{\mathrm{z}}$ and $H_{\mathrm{r}}$ ) must be satisfied. Equations are formulated and solved from these boundary and interface conditions to determine the unknown coefficients and the magnetic vector potentials of each region. The magnetic vector potential of a multi-turn coil as shown in Fig.2.b) can be obtained by integrating the magnetic vector potential of a filamentary coil $A_{\text {filamentary }}\left(r, z, r_{0}, z_{0}\right)$ along the rectangular cross section of a multi-turn coil [15].

$$
A(r, z)=\int_{r_{1}}^{r_{2}} \int_{z_{1}}^{z_{2}} A_{\text {filamentary }}\left(r, z, r_{0}, z_{0}\right) d r_{0} d z_{0}
$$

The magnetic potential $A_{2-3}(r, z)$ of region 2-3 in Fig.2.b) can be obtained by substituting $z$ for $z_{2}$ in $A_{2}(r, z)$ and $z$ for $z_{1}$ in $A_{3}(r, z)$, and then adding them together.
The expression for the I-core coil impedance above the twolayer conductor is finally obtained by integrating the magnetic vector potential $A_{2-3}(r, z)$ over the cross section of the coil.

$$
\begin{aligned}
& Z=\frac{j \omega 2 \pi N}{I\left(z_{2}-z_{1}\right)\left(r_{2}-r_{1}\right)} \int_{r_{1}}^{r_{2} z_{2}} \int_{z_{1}} r A_{2-3}(r, z) d r d z \\
& =\frac{j \omega \mu \pi N^{2}}{\left(r_{2}-r_{1}\right)^{2}\left(z_{2}-z_{1}\right)^{2}} \chi\left(\mathbf{p} r_{1}, \mathbf{p} r_{2}\right) \mathbf{p}^{-4}\left\langle 2\left(z_{2}-z_{1}\right) \mathbf{p}\right. \\
& \left.+e^{\mathbf{p}\left(z_{1}-z_{2}\right)}-e^{\mathbf{p}\left(z_{2}-z_{1}\right)}+\mathbf{W}_{1} \mathbf{W}_{2}^{-1} \mathbf{W}_{3}\right\rangle \mathbf{D}^{-1} \chi\left(\mathbf{p} r_{1}, \mathbf{p} r_{2}\right) \mathbf{p}^{-3}
\end{aligned}
$$

where $I$ is the current carried in each wire loop.

$$
\begin{aligned}
& \chi\left(x_{1}, x_{2}\right)=\int_{x_{1}}^{x_{2}} x R_{1}(x) d x \\
& \mathbf{W}_{1}=\left(e^{-\mathbf{p} z_{1}}-e^{-\mathbf{p} z_{2}}\right) \mathbf{C}_{37}-\left(e^{\mathbf{p} z_{2}}-e^{\mathbf{p} z_{1}}\right) \mathbf{B}_{37} \\
& \mathbf{W}_{2}=(\mathbf{T}-\mathbf{U}) e^{-\mathbf{p} h_{1}} \mathbf{C}_{37}-(\mathbf{T}+\mathbf{U}) e^{\mathbf{p} h_{1}} \mathbf{B}_{37} \\
& \mathbf{W}_{3}=(\mathbf{T}+\mathbf{U})\left(e^{\mathbf{p}\left(h_{1}-z_{1}\right)}-e^{\mathbf{p}\left(h_{1}-z_{2}\right)}\right) \\
& -(\mathbf{T}-\mathbf{U})\left(e^{\mathbf{p}\left(z_{2}-h_{1}\right)}-e^{\mathbf{p}\left(z_{1}-h_{1}\right)}\right) \\
& \mathbf{C}_{37}=\frac{1}{2}\left[\left(\mathbf{H}^{-1}+\mathbf{N}^{-1}\right) \mathbf{E} \mathbf{C}_{47}+\left(-\mathbf{H}^{-1}+\mathbf{N}^{-1}\right) \mathbf{E} \mathbf{B}_{47}\right] \\
& \mathbf{B}_{37}=\frac{1}{2}\left[\left(-\mathbf{H}^{-1}+\mathbf{N}^{-1}\right) \mathbf{E} \mathbf{C}_{47}+\left(\mathbf{H}^{-1}+\mathbf{N}^{-1}\right) \mathbf{E} \mathbf{B}_{47}\right] \\
& \mathbf{C}_{47}=\frac{1}{2} e^{-\mathbf{q} d_{1}}\left[\left(1+\mathbf{q} \mathbf{m}^{-1}\right) e^{\mathbf{m} d_{1}} \mathbf{C}_{57}+\left(1-\mathbf{q} \mathbf{m}^{-1}\right) e^{-\mathbf{m} d_{1}} \mathbf{B}_{57}\right] \\
& \mathbf{B}_{47}=\frac{1}{2} e^{\mathbf{q} d_{1}}\left[\left(1-\mathbf{q m}^{-1}\right) e^{\mathbf{m} d_{1}} \mathbf{C}_{57}+\left(1+\mathbf{q} \mathbf{m}^{-1}\right) e^{-\mathbf{m} d_{1}} \mathbf{B}_{57}\right] \\
& \mathbf{C}_{57}=\frac{1}{2} e^{-\mathbf{m} d_{2}}\left[\left(\mathbf{E}^{-1} \mu_{5} \mathbf{K}+\mathbf{m} \mathbf{q}^{-1} \mathbf{E}^{-1} \mathbf{V}\right) e^{\mathbf{u} d_{2}} \mathbf{C}_{67}\right. \\
& \left.+\left(\mathbf{E}^{-1} \mu_{5} \mathbf{K}-\mathbf{m q}^{-1} \mathbf{E}^{-1} \mathbf{V}\right) e^{-\mathbf{u d} d_{2}} \mathbf{B}_{67}\right] \\
& \mathbf{B}_{57}=\frac{1}{2} e^{\mathbf{m} d_{2}}\left[\left(\mathbf{E}^{-1} \mu_{5} \mathbf{K}-\mathbf{m q}^{-1} \mathbf{E}^{-1} \mathbf{V}\right) e^{\mathbf{u} d_{2}} \mathbf{C}_{67}\right. \\
& \left.+\left(\mathbf{E}^{-1} \mu_{5} \mathbf{K}+\mathbf{m q}^{-1} \mathbf{E}^{-1} \mathbf{V}\right) e^{-\mathbf{u} d_{2}} \mathbf{B}_{67}\right] \\
& \mathbf{C}_{67}=\frac{1}{2} e^{-\mathbf{u} d_{3}}\left(\mathbf{V}^{-1}-\mathbf{K}^{-1}\right) \mathbf{E} e^{-\mathbf{q} d_{3}} \\
& \mathbf{B}_{67}=-\frac{1}{2} e^{\mathbf{u} d_{3}}\left(\mathbf{V}^{-1}+\mathbf{K}^{-1}\right) \mathbf{E} e^{-\mathbf{q} d_{3}}
\end{aligned}
$$

where

$$
\begin{gathered}
\mathbf{C}_{n 7}=\frac{\mathbf{C}_{n}}{\mathbf{B}_{7}} \\
\mathbf{B}_{n 7}=\frac{\mathbf{B}_{n}}{\mathbf{B}_{7}}
\end{gathered}
$$


Where $\mathbf{T}, \mathbf{U}, \mathbf{H}, \mathbf{N}, \mathbf{K}$, and $\mathbf{V}$ are full matrices. $\mathbf{E}$ and $\mathbf{D}$ are diagonal matrices, they are defined in the Appendix.

\section{RESULTS AND DISCUSSION}

The impedance $Z=R+j X$ of an I-core coil located above the infinite plane conductor with the conductive cylinder below was calculated using Mathematica according to (21). The parameters of the coil and conductive material used in the calculation are shown in Table 1.

When the conductor is absent $\left(\sigma_{5}=\sigma_{6}=0\right)$, the whole problem region is reduced into four regions. The expression of the magnetic vector potential of region four changes to the following form:

$$
A_{4}(r, z)=J_{1}\left(\mathbf{q}^{\mathbf{T}} r\right) \mathbf{q}^{-1} e^{\mathbf{q} z} \mathbf{B}_{4}
$$

In this case, the coil impedance can also be calculated using (21), and the coil impedance is expressed as $Z_{0}=R_{0}+j X_{0}, \mathrm{~W}_{1}$ and $\mathrm{W}_{2}$ change into the following forms:

$$
\begin{aligned}
& \mathbf{W}_{1}=\left(e^{-\mathbf{p} z_{1}}-e^{-\mathbf{p} z_{2}}\right) \mathbf{C}_{34}-\left(e^{\mathbf{p} z_{2}}-e^{\mathbf{p} z_{1}}\right) \mathbf{B}_{34} \\
& \mathbf{W}_{2}=(\mathbf{T}-\mathbf{U}) e^{-\mathbf{p} h_{1}} \mathbf{C}_{34}-(\mathbf{T}+\mathbf{U}) e^{\mathbf{p} h_{1}} \mathbf{B}_{34}
\end{aligned}
$$

where

$$
\begin{gathered}
\mathbf{C}_{34}=\frac{\mathbf{C}_{3}}{\mathbf{B}_{4}}=\frac{1}{2}\left(\mathbf{H}^{-1}-\mathbf{N}^{-1}\right) \mathbf{E} \\
\mathbf{B}_{34}=\frac{\mathbf{B}_{3}}{\mathbf{B}_{4}}=-\frac{1}{2}\left(\mathbf{H}^{-1}+\mathbf{N}^{-1}\right) \mathbf{E}
\end{gathered}
$$

Table 1. The parameters of the coil, core and conductor used in analytical calculations and FEM.

\begin{tabular}{|c|c|c|}
\hline Inner coil radius & $r_{1}$ & $5 \mathrm{~mm}$ \\
\hline Outer coil radius & $r_{2}$ & $8 \mathrm{~mm}$ \\
\hline Offset & $z_{1}$ & $0 \mathrm{~mm}$ \\
\hline Parameter & $z_{2}$ & $7 \mathrm{~mm}$ \\
\hline Number of turns & $N$ & 800 \\
\hline Core relative permeability & $\mu_{f}$ & 500 \\
\hline Core radius & $a_{1}$ & $4.5 \mathrm{~mm}$ \\
\hline Core height & $h_{1}$ & $8 \mathrm{~mm}$ \\
\hline Liftoff & $d_{1}$ & $0.2 \mathrm{~mm}$ \\
\hline Parameter & $d_{2}$ & $0.4 \mathrm{~mm}$ \\
\hline Parameter & $d_{3}$ & $5.4 \mathrm{~mm}$ \\
\hline Conductive cylinder radius & $c$ & $15 \mathrm{~mm}$ \\
\hline Relative permeability & $\mu_{5} \mu_{6}$ & 1 \\
\hline Conductivity & $\sigma_{5}$ & $10 \mathrm{MS} / \mathrm{m}$ \\
\hline Conductivity & $\sigma_{6}$ & $16 \mathrm{MS} / \mathrm{m}$ \\
\hline Truncated domain radius & $b$ & $60 \mathrm{~mm}$ \\
\hline Summation terms & $N_{s}$ & 60 \\
\hline
\end{tabular}

When the probe is placed over an infinite plane conductor with the conducting cylinder below and with the two-layer conductor removed, the change in coil impedance is expressed as $\Delta Z=\Delta R+j \Delta X=Z-Z_{0}$. Specifically, the changes in resistance and reactance are expressed as $\Delta R=R-R_{0}$ and
$\Delta X=X-X_{0}$, respectively. When the coil excitation frequency varies from $0.1 \mathrm{kHz}$ to $10 \mathrm{kHz}$, the impedance changes of the I-core coil are calculated respectively.

The analytical calculation results were compared with the results of the finite element method (FEM) using the ANSYS Maxwell software. In the FEM, an adaptive mesh refinement method is used to increase the number of meshes on the surface of the coil and conductor, so as to improve the accuracy of simulated results.

Fig.3.a) and Fig.3.b) show the real and imaginary parts of the I-core coil absolute impedance change with and without the two-layer conductor as a function of frequency, obtained from analytical calculation and the finite element method, respectively. It can be seen from Fig.3.a) that the absolute resistance variation of I-core coil and air-core coil increases with the increase of frequency. Excitation by the same frequency, the absolute values of resistance and reactance change of I-core coil are greater than those of air-core coil. The absolute impedance variation obtained by the TREE method is in good agreement with the results obtained by FEM. The maximum relative error between analytical calculation and FEM is not more than $1.0 \%$.

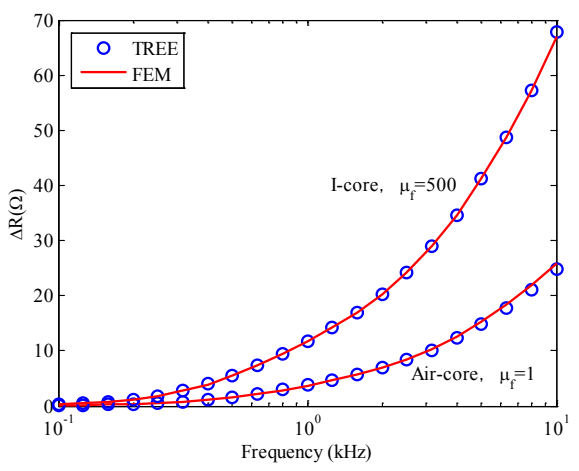

a)

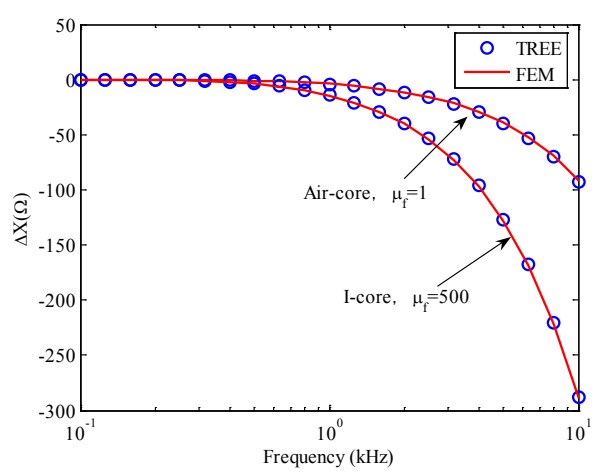

b)

Fig.3. a) Real part and b) Imaginary part of the absolute impedance change with and without the two-layer conductor as a function of frequency for an air-core coil $\left(\mu_{\mathrm{f}}=1\right)$ and an I-core coil $\left(\mu_{\mathrm{f}}=500\right)$.

The influence of the conductive cylinder below the infinite plane conductor on the variation of coil impedance depends on many factors, such as dimensions of conductive cylinder, conductor characteristics and probe parameters. In order to detect the conducting cylinder below the plane conductor, the 
analytical model should be capable of calculating the impedance change caused by the conducting cylinder.

When an I-core coil is located above the infinite plane conductor without the conductive cylinder, the whole region is divided into six regions, the expression of the magnetic vector potential of region six changes to the following form:

$$
A_{6}(r, z)=J_{1}\left(\mathbf{q}^{\mathrm{T}} r\right) \mathbf{q}^{-1} e^{\mathbf{q} z} \mathbf{B}_{6}
$$

The impedance of the I-core coil can also be calculated using (21), and the coil impedance is $Z_{\mathrm{c}}=R_{\mathrm{c}}+j X_{\mathrm{c}}$, in this case, $\mathrm{W}_{1}$ and $\mathrm{W}_{2}$ change into the following forms:

$$
\begin{aligned}
& \mathbf{W}_{1}=\left(e^{-\mathbf{p} z_{1}}-e^{-\mathbf{p} z_{2}}\right) \mathbf{C}_{36}-\left(e^{\mathbf{p} z_{2}}-e^{\mathbf{p} z_{1}}\right) \mathbf{B}_{36} \\
& \mathbf{W}_{2}=(\mathbf{T}-\mathbf{U}) e^{-\mathbf{p} h_{1}} \mathbf{C}_{36}-(\mathbf{T}+\mathbf{U}) e^{\mathbf{p} h_{1}} \mathbf{B}_{36}
\end{aligned}
$$

where

$$
\begin{gathered}
\mathbf{C}_{36}=\frac{1}{2}\left[\left(\mathbf{H}^{-1}+\mathbf{N}^{-1}\right) \mathbf{E} \mathbf{C}_{46}+\left(-\mathbf{H}^{-1}+\mathbf{N}^{-1}\right) \mathbf{E} \mathbf{B}_{46}\right] \\
\mathbf{B}_{36}=\frac{1}{2}\left[\left(-\mathbf{H}^{-1}+\mathbf{N}^{-1}\right) \mathbf{E} \mathbf{C}_{46}+\left(\mathbf{H}^{-1}+\mathbf{N}^{-1}\right) \mathbf{E} \mathbf{B}_{46}\right] \\
\mathbf{C}_{46}=\frac{1}{2} e^{-\mathbf{q} d_{1}}\left[\left(1+\mathbf{q} \mathbf{m}^{-1}\right) e^{\mathbf{m} d_{1}} \mathbf{C}_{56}+\left(1-\mathbf{q} \mathbf{m}^{-1}\right) e^{-\mathbf{m} d_{1}} \mathbf{B}_{56}\right] \\
\mathbf{B}_{46}=\frac{1}{2} e^{\mathbf{q} d_{1}}\left[\left(1-\mathbf{q} \mathbf{m}^{-1}\right) e^{\mathbf{m} d_{1}} \mathbf{C}_{56}+\left(1+\mathbf{q} \mathbf{m}^{-1}\right) e^{-\mathbf{m} d_{1}} \mathbf{B}_{56}\right] \\
\mathbf{C}_{56}=\frac{1}{2} e^{-\mathbf{m} d_{2}}\left(\mathbf{m} \mathbf{q}^{-1}-\mu_{5}\right) e^{-\mathbf{q} d_{2}} \\
\mathbf{B}_{56}=-\frac{1}{2} e^{\mathbf{m} d_{2}}\left(\mu_{5}+\mathbf{m q} \mathbf{q}^{-1}\right) e^{-\mathbf{q} d_{2}}
\end{gathered}
$$

where

$$
\begin{gathered}
\mathbf{C}_{n 6}=\frac{\mathbf{C}_{n}}{\mathbf{B}_{6}} \\
\mathbf{B}_{n 6}=\frac{\mathbf{B}_{n}}{\mathbf{B}_{6}}
\end{gathered}
$$

The changes in coil resistance and reactance due to the presence of conductive cylinder are expressed as $\Delta R_{\mathrm{c}}=R-R_{\mathrm{c}}$ and $\Delta X_{\mathrm{c}}=X-X_{\mathrm{c}}$. Fig.4.a) and Fig.4.b) show the absolute coil resistance variation $\Delta R_{\mathrm{c}}$ and reactance variation $\Delta X_{\mathrm{c}}$ in relation to different excitation frequency.

It can be seen from Fig.4.a) that when the excitation frequency is about $4 \mathrm{kHz}$, the I-core coil and the air-core coil have the same real part of absolute impedance variation. At the same frequency, the absolute value of the imaginary part of impedance change of I-core coil shown in Fig.4.b) is larger than that of the air-core coil.

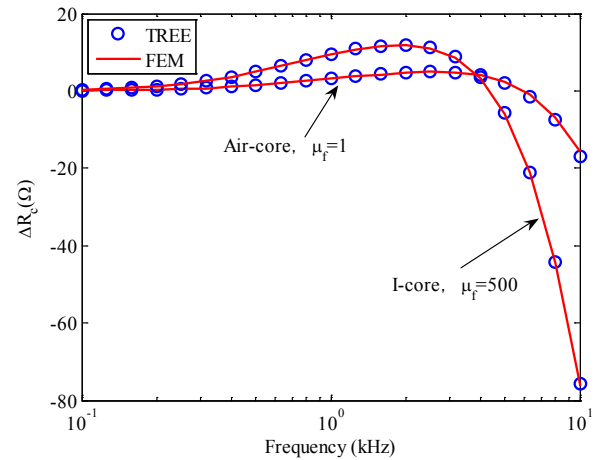

a)

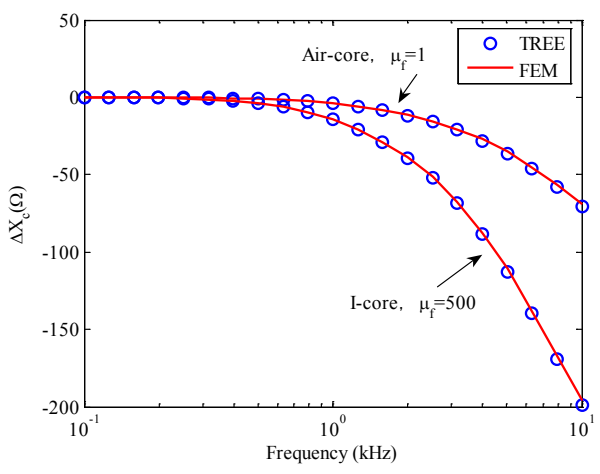

b)

Fig.4. a) Real part and b) Imaginary part of the absolute impedance change with and without the conductive cylinder as a function of frequency for an air-core coil $\left(\mu_{\mathrm{f}}=1\right)$ and an I-core coil $\left(\mu_{\mathrm{f}}=500\right)$.

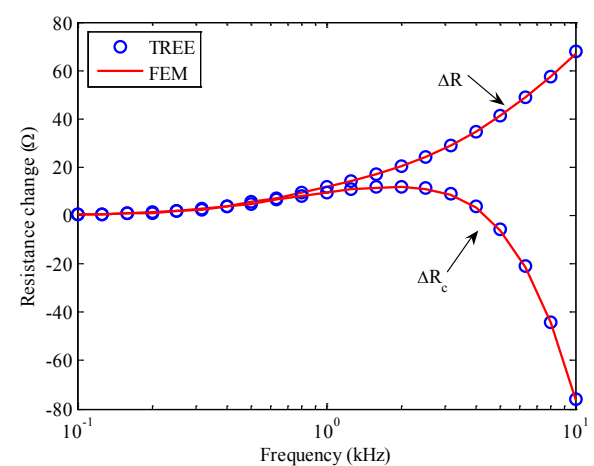

a)

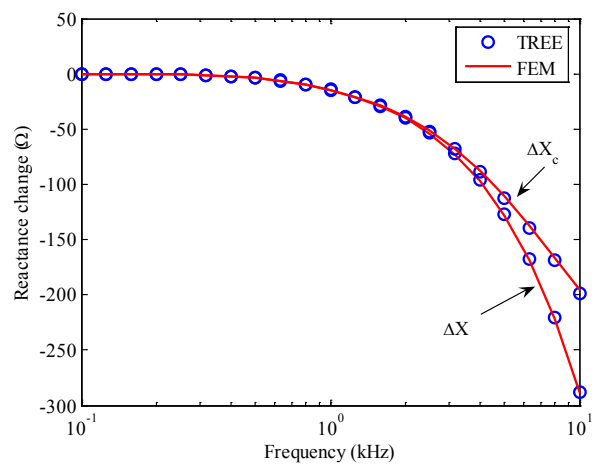

b)

Fig.5. a) Real part and b) Imaginary part of the absolute impedance changes as a function of frequency for I-core coil. 
Fig.5.a) and Fig.5.b) show the real and imaginary parts of the absolute impedance variation of the I-core coil at different frequencies, caused by the presence or absence of the conductive cylinder and the presence or absence of the twolayer conductor at the same excitation frequency.

It can be seen from Fig.5.a), when the frequency is less than $0.8 \mathrm{kHz}$, real parts of the absolute impedance changes of the two cases are nearly the same. When the frequency is greater than $0.8 \mathrm{kHz}$, real part of the absolute coil impedance change caused by the two-layer conductor is greater than that caused by the conductive cylinder.

Fig.5.b) shows that when the frequency is less than $2.5 \mathrm{kHz}$, the imaginary part of absolute impedance changes is basically the same, but when the frequency is greater than $2.5 \mathrm{kHz}$, the absolute value of imaginary part of impedance changes caused by the two-layer conductor is greater than that caused by the conductive cylinder.

\section{EXPERIMENTAL VERIFICATION}

Experiments were also set up to verify the expression of impedance for an I-core coil in (21). An I-core coil, conductive cylinder and plane conductor are shown in Fig.6.a) and Fig.6.b), respectively. The parameters of the coil, core and conductors used in experiments are shown in Table 2.

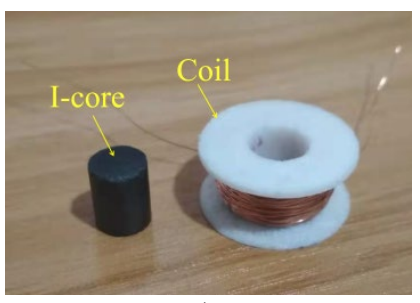

a)

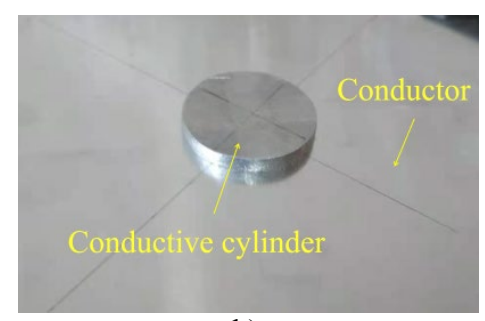

b)

Fig.6. a) I-core, coil and b) conductors used in experiments.

The experimental configuration is shown in Fig.7. The unknown impedance of the coil is calculated by measuring the voltage and current values across the coil. The current is obtained by measuring the voltage drop across a precision resistor of $1 \mathrm{k} \Omega$ in series with the coil. A Tektronix AFG3021C function generator is used to provide sine wave excitation of different frequencies to the coil. The AFG3021C's bandwidth of $25 \mathrm{MHz}$ is well-suited for this measurement. The voltages and phase difference before and after the $1 \mathrm{k} \Omega$ precision resistor are measured by a Tektronix DPO2012B oscilloscope. The DPO2012B's DC gain accuracy is $\pm 3 \%$ at a $10 \mathrm{mV} / \mathrm{div}$ to $5 \mathrm{~V} / \mathrm{div}$ setting. The results of impedance change achieved by experiments and analytical calculation at nine different frequencies are compared in Table 3 . The results match very well, and they verify the correctness of the analytical model.

Table 2. The parameters of the coil, core and conductors used in experiments and analytical calculations.

\begin{tabular}{|c|c|c|}
\hline Inner coil radius & $r_{1}$ & $5.2 \mathrm{~mm}$ \\
\hline outer coil radius & $r_{2}$ & $7.2 \mathrm{~mm}$ \\
\hline Offset & $z_{1}$ & $1.2 \mathrm{~mm}$ \\
\hline Parameter & $z_{2}$ & $9.2 \mathrm{~mm}$ \\
\hline Number of turns & $N$ & 387 \\
\hline Core relative permeability & $\mu_{f}$ & 2000 \\
\hline Core radius & $a_{1}$ & $4 \mathrm{~mm}$ \\
\hline Core height & $h_{1}$ & $10.5 \mathrm{~mm}$ \\
\hline Liftoff & $d_{1}$ & $0.1 \mathrm{~mm}$ \\
\hline Parameter & $d_{2}$ & $0.6 \mathrm{~mm}$ \\
\hline Parameter & $d_{3}$ & $4.6 \mathrm{~mm}$ \\
\hline Conductive cylinder radius & $c$ & $15 \mathrm{~mm}$ \\
\hline Relative permeability & $\mu_{5}, \mu_{6}$ & 1 \\
\hline Conductivity & $\sigma_{5}$ & $36 \mathrm{MS} / \mathrm{m}$ \\
\hline Conductivity & $\sigma_{6}$ & $36 \mathrm{MS} / \mathrm{m}$ \\
\hline Truncated domain radius & $b$ & $60 \mathrm{~mm}$ \\
\hline Summation terms & $N s$ & 60 \\
\hline
\end{tabular}

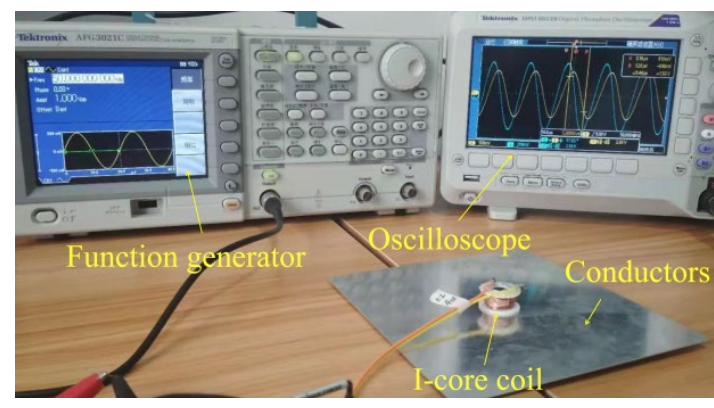

Fig.7. Experimental setup.

Table 3. The impedance changes of I-core coil located above a twolayer conductor and in the air obtained by the TREE method and experiments.

\begin{tabular}{|c|c|c|}
\hline \multirow{2}{*}{$\begin{array}{c}\text { Frequency } \\
\text { (kHz) }\end{array}$} & \multicolumn{2}{|c|}{$\Delta \mathbf{Z}[\mathbf{O h m}]$} \\
\cline { 2 - 3 } & TREE & Experiment \\
\hline 10 & $8.39-60.64 i$ & $8.23-59.39 i$ \\
\hline 15 & $10.59-93.61 i$ & $10.60-94.06 i$ \\
\hline 20 & $12.44-126.93 i$ & $12.78-128.63 i$ \\
\hline 25 & $14.08-160.47 i$ & $13.96-161.53 i$ \\
\hline 30 & $15.56-194.17 i$ & $15.41-202.69 i$ \\
\hline 35 & $16.93-228.02 i$ & $16.82-213.70 i$ \\
\hline 40 & $18.20-261.94 i$ & $18.07-262 i$ \\
\hline 45 & $19.40-295.91 i$ & $19.23-306.18 i$ \\
\hline 50 & $20.53-330.02 i$ & $20.59-333.61 i$ \\
\hline
\end{tabular}

\section{CONCLUSION}

In this paper, an analytical model for calculation of the impedance of an I-core coil placed above an infinite plate conductor and a conducting cylinder is presented. A closed 
form impedance expression of the I-core coil is derived by the TREE method. The calculation of coil impedance is performed in Mathematica software, and the calculation accuracy can be controlled by changing the truncated solution domain and the summation terms. The analytical results are in good agreement with the finite element method and the experimental results. The proposed analytical model can be applied to design eddy current probes, simulation of defect detection in ECT, and it can also be directly applied in the evaluation of multi-layer conductor.

\section{APPENDIX}

$$
\begin{gathered}
\mathbf{E}=\int_{0}^{b} r J_{0}(\mathbf{q} r) J_{0}\left(\mathbf{q}^{\mathrm{T}} r\right) d r=\int_{0}^{b} r J_{1}(\mathbf{q} r) J_{1}\left(\mathbf{q}^{\mathrm{T}} r\right) d r \\
\mathbf{T}=\int_{0}^{a_{1}} r J_{0}(\mathbf{q} r) J_{0}\left(\mathbf{p}^{\mathrm{T}} r\right) d r+\int_{a_{1}}^{b} r J_{0}(\mathbf{q} r) R_{0}\left(\mathbf{p}^{\mathrm{T}} r\right) d r \\
\mathbf{U}=\frac{1}{\mu_{f}} \int_{0}^{a_{1}} r J_{1}(\mathbf{q} r) J_{1}\left(\mathbf{p}^{\mathrm{T}} r\right) d r+\int_{a_{1}}^{b} r J_{1}(\mathbf{q} r) R_{1}\left(\mathbf{p}^{\mathrm{T}} r\right) d r \\
\mathbf{D}=\frac{1}{\mu_{f}} \int_{0}^{a_{1}} r J_{1}(\mathbf{p} r) J_{1}\left(\mathbf{p}^{\mathrm{T}} r\right) d r+\int_{a_{1}}^{b} r R_{1}(\mathbf{p} r) R_{1}\left(\mathbf{p}^{\mathrm{T}} r\right) d r \\
\mathbf{H}=\int_{0}^{a_{1}} r J_{0}(\mathbf{q} r) J_{0}\left(\mathbf{p}^{\mathbf{T}} r\right) d r+\int_{a_{1}}^{b} r J_{0}(\mathbf{q} r) R_{0}\left(\mathbf{p}^{\mathrm{T}} r\right) d r \\
\mathbf{N}=\frac{1}{\mu_{f}} \int_{0}^{a_{1}} r J_{1}(\mathbf{q} r) J_{1}\left(\mathbf{p}^{\mathrm{T}} r\right) d r+\int_{a_{1}}^{b} r J_{1}(\mathbf{q} r) R_{1}\left(\mathbf{p}^{\mathrm{T}} r\right) d r \\
\mathbf{V}=F_{1}(\mathbf{u} c) \mathbf{v} \mathbf{u}^{-1} \int_{0}^{c} r J_{0}(\mathbf{v} r) J_{0}\left(\mathbf{q}^{\mathrm{T}} r\right) d r+J_{1}(\mathbf{v} c) \int_{c}^{b} r F_{0}(\mathbf{u} r) J_{0}\left(\mathbf{q}^{\mathrm{T}} r\right) d r \\
\mathbf{K}=\frac{1}{\mu_{6}} F_{1}(\mathbf{u} c) \int_{0}^{c} r J_{1}(\mathbf{v} r) J_{1}\left(\mathbf{q}^{\mathrm{T}} r\right) d r+J_{1}(\mathbf{v} c) \int_{c}^{b} r F_{1}(\mathbf{u} r) J_{1}\left(\mathbf{q}^{\mathrm{T}} r\right) d r
\end{gathered}
$$

\section{REFERENCES}

[1] Lu, Y., Bowler, J.R., Theodoulidis, T.P. (2012). An analytical model of a ferrite-cored inductor used as an eddy current probe. Journal of Applied Physics, 111 (10), 103907-10.

[2] Zhang, S. (2020). an analytical model of a new T-cored coil used for eddy current nondestructive evaluation. Applied Computational Electromagnetics Society Journal, 35 (9), 1099-1104.

[3] Tytko, G., Dziczkowski, L. (2018). Fast calculation of the filamentary coil impedance using the truncated region eigenfunction expansion method. Applied Computational Electromagnetics Society Journal, 33 (12), 1461-1466.
[4] Theodoulidis, T.P., Kriezis, E.E. (2005). Series expansions in eddy current nondestructive evaluation models. Journal of Materials Processing Technology, 161 (1-2), 343-347.

[5] Bayani, H., Theodoulidis, T.P., Sasada, I. (2007). Application of eigenfunction expansions to eddy current NDE: A model of cup-cored probes. In Studies in Applied Electromagnetics and Mechanics. IOS Press, 57-64. ISBN 978-158603752-9.

[6] Sakkaki, F., Bayani, H. (2012). Solution to the problem of E-cored coil above a layered half-space using the method of truncated region eigenfunction expansion. Journal of Applied Physics, 111 (7), 2829-64.

[7] Theodoulidis, T.P. (2003). Model of ferrite-cored probes for eddy current nondestructive evaluation. Journal of Applied Physics, 93 (5), 3071-3078.

[8] Tytko, G., Dziczkowski, L. (2017). An analytical model of an I-cored coil with a circular air gap. IEEE Transactions on Magnetics, 53 (4), 620104.

[9] Tytko, G., Dziczkowski, L. (2015). E-cored coil with a circular air gap inside the core column used in eddy current testing. IEEE Transactions on Magnetics, 51 (9), $1-4$.

[10] Tytko, G., Dziczkowski, L. (2018). I-cored coil probe located above a conductive plate with a surface hole. Measurement Science Review, 18 (1), 7-12.

[11] Koliskina, V. (2014). Calculation of eigenvalues for eddy current testing problems. Boundary Field Problems and Computer Simulation, 53 (1), 9-11.

[12] Theodoulidis, T.P., Kriezis, E.E. (2006). Eddy Current Canonical Problems (With Applications to Nondestructive Evaluation). Tech Science Press, 106121. ISBN 978-0971788015.

[13] Theodoulidis, T.P., Bowler, J.R. (2005). Eddy-current interaction of a long coil with a slot in a conductive plate. IEEE Transactions on Magnetics, 41 (4), 12381247.

[14] Tytko, G., Dawidowski, L. (2019). Locating complex eigenvalues for analytical eddy-current models used to detect flaws. COMPEL - The International Journal for Computation and Mathematics in Electrical and Electronic Engineering, 38 (6), 1800-1809.

[15] Dodd, C.V., Deeds, W.E. (1968). Analytical solutions to eddy-current probe-coil problems. Journal of Applied Physics, 39 (6), 2829-2838.

Received March 14, 2021 Accepted July 12, 2021 